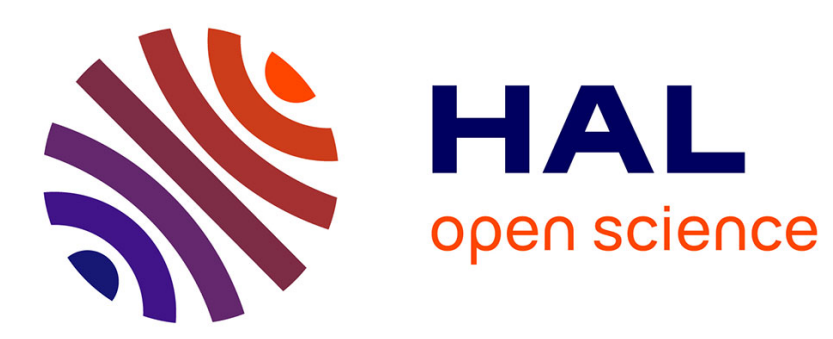

\title{
Choosing a thermal model for electrothermal simulation of power semiconductor devices
}

\author{
Anis Ammous, Sami Ghedira, Bruno Allard, Hervé Morel, Denise Renault
}

\section{To cite this version:}

Anis Ammous, Sami Ghedira, Bruno Allard, Hervé Morel, Denise Renault. Choosing a thermal model for electrothermal simulation of power semiconductor devices. IEEE Transactions on Power Electronics, 1999, 14 (2), pp.300-307. hal-00140099

\section{HAL Id: hal-00140099 \\ https://hal.science/hal-00140099}

Submitted on 6 Apr 2007

HAL is a multi-disciplinary open access archive for the deposit and dissemination of scientific research documents, whether they are published or not. The documents may come from teaching and research institutions in France or abroad, or from public or private research centers.
L'archive ouverte pluridisciplinaire HAL, est destinée au dépôt et à la diffusion de documents scientifiques de niveau recherche, publiés ou non, émanant des établissements d'enseignement et de recherche français ou étrangers, des laboratoires publics ou privés. 


\title{
Choosing a Thermal Model for Electrothermal Simulation of Power Semiconductor Devices
}

\author{
Anis Ammous, Sami Ghedira, Bruno Allard, Member IEEE, Hervé Morel, and Denise Renault
}

\begin{abstract}
The literature proposes some thermal models needed for the electrothermal simulation of power electronic systems. This paper gives a useful analysis about the choice of the thermal model circuit networks, equivalent to a discretization of the heat equation by the finite difference method (FDM) and the finiteelement method (FEM), and an analytic model developed by applying an internal approximation of the heat diffusion problem. The effect of the boundary condition representation and the introduced errors on temperature response at the heat source are studied. This study is advantageous, particularly for large surges of a short time duration.
\end{abstract}

Index Terms - Finite difference method, finite-element method, large surges, thermal circuit networks, thermal model.

\begin{tabular}{|c|c|}
\hline & NOMENCLATURE \\
\hline$L(\mathrm{~cm}), S\left(\mathrm{~cm}^{2}\right)$ & $\begin{array}{l}\text { Effective length and area of the semi- } \\
\text { conductor device. }\end{array}$ \\
\hline$c$ & Silicon-specific heat $(\mathrm{J} / \mathrm{g} / \mathrm{K})$. \\
\hline$\rho$ & Silicon mass density $\left(\mathrm{g} / \mathrm{cm}^{3}\right)$ \\
\hline$T$ & Absolute temperature $(\mathrm{K})$. \\
\hline & Input temperature $(\mathrm{K})$. \\
\hline$P_{\mathrm{in}}(t)$ & Input dissipated power (W). \\
\hline$K$ & Thermal conductivity $(\mathrm{W} / \mathrm{cm} / \mathrm{K})$. \\
\hline
\end{tabular}

\section{INTRODUCTION}

$\mathbf{E}$ QUIVALENT electrical circuits as thermal modeling are largely used because of their easy implementation in circuit simulators in which most of the semiconductor device models are implemented. This enables a simple coupling between electrical and thermal phenomena.

Semiconductor manufacturers provide the "effective transient thermal impedance curve" [1] as the key tool to calculate the peak junction temperature in the device. This curve does not include dissipated power for pulse width in the order of few microseconds. This curve is based on classical analysis of the temperature evolution in the structure, which assumes a semifinite solid. It is obtained from a convolution method. $S$. Clement in [2] evokes this problem and proposes a correction of the calculated temperature obtained by the analytical formulation developed by the classical method to take into account the finite dimension of the die. This new analysis gives an appropriate correction of the estimated peak junction

Manuscript received November 12, 1997; revised July 13, 1998. Recommended by Associate Editor, W. Portnoy.

The authors are with CEGELY-INSA Lyon, F-69621 Villeurbanne Cedex, France.

Publisher Item Identifier S 0885-8993(99)01830-X.

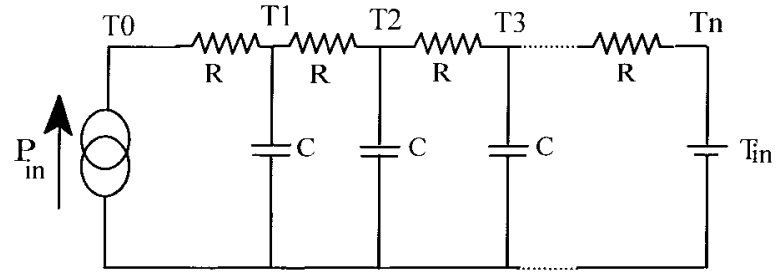

Fig. 1. Equivalent thermal circuit networks obtained by the FDM.

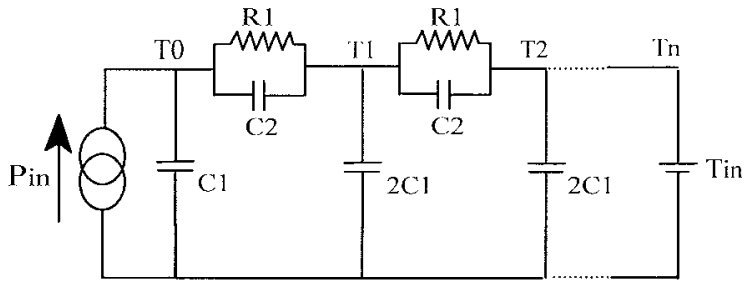

Fig. 2. Equivalent thermal circuit networks obtained by the FEM

temperature of semiconductor devices useful in the case of large surges of short time duration.

Unfortunately, for a given input power dissipation, the peak junction temperature is given by a convolution model, and it does not fit with circuit simulators.

On the other hand, equivalent electrical circuits and analytical model fit well with circuit simulators. Classical equivalent circuits (Fig. 1) are based on a finite difference discretization of the heat equation [3], [4]. A recent paper [5] proposed another equivalent electrical circuit based on the finite element approach (Fig. 2). In [6], the authors proposed an analytical model based on internal approximation. In fact, with a suitable choice of the decomposition functions, this method gives a good tradeoff between accuracy and simulation time.

The present paper gives an analysis of the three methods, the finite difference method (FDM), the finite-element method (FEM), and the analytical model (IAAM). Especially, the accuracy of these methods is treated for large surges of a short time duration, where the gap between the estimated temperature predicted by the methods is not negligible. This corresponds to the practical case of most of the power device operations.

In Section II, the FDM is recalled and the error on estimated temperature responses is studied. The FEM results are presented in the third section. In the fourth section, the results given by the IAAM model are presented and compared to the other models. 
In the case of a vertical power device, where the thickness $L$ is small compared to other dimensions, heat is generated at the top surface of silicon and flows linearly along the $x$ axes (perpendicular to the silicon surface $S$ ). So, the top surface is considered to be a geometrical boundary of the device at $x=$ 0 , where the input power $P_{\mathrm{in}}(t)$ is assumed to be uniformly dissipated. The lower surface (at $x=L$ ) is considered to be the cooling boundary, where the temperature is assumed to be equal to the input temperature $T_{\mathrm{in}}$. Convection and radiation are assumed to be negligible. So, a one-dimensional (1-D) heat flow may be considered, and since the thermal characteristics of silicon are assumed to be independent of temperature, the thermal system is governed by the following partial differential equation:

$$
K \frac{\partial^{2} T}{\partial x^{2}}=\rho c \frac{\partial T}{\partial t}
$$

with boundary conditions

$$
\begin{aligned}
& \left.S K \frac{\partial T}{\partial x}\right]_{x=0}=-P_{\mathrm{in}}(t) \\
& T(t, x=L)=T_{\mathrm{in}}(t) .
\end{aligned}
$$

We note that this analysis corresponds also to the case of microelectronic integrated circuit (IC), where the distribution of the heat generation at the top of the silicon chip is uniform.

\section{Finite Difference Method}

The 1-D structure is geometrically discretized using $n$ constant steps of value $h(\mathrm{~cm})$. At node number $i$, the discretization of (1) with a constant step value $h$ gives the classical relation [3]

$$
\frac{T_{i+1}(t)+T_{i-1}(t)-2 T_{i}(t)}{h^{2}}=\frac{\rho c}{K} \frac{d T_{i}(t)}{d t}, \quad i=1, n-1
$$

where $T_{i}$ is the temperature at the node number $i$ and $h=L / n$.

The first-order discretization of the boundary condition at $x=0$ gives

$$
\frac{T_{0}(t)-T_{1}(t)}{h}=\frac{P_{\mathrm{in}}(t)}{K S} .
$$

To take into account the boundary condition at $x=L$, (3) has to be considered with $T_{n}=T_{\mathrm{in}}$.

Taking into account (3) and (4), the equivalent circuit of the 1-D thermal model is shown in Fig. 1, where $R=$ $(h / K S)(K / W)$ and $C=h \rho c S(J / K)$ are the elementary thermal resistance and capacitance, respectively.

For a power pulse of high magnitude and very short duration (pulse duration less than the temperature response time in the silicon die), the FDM introduces an important error, especially on the first node temperature $T_{0}$. This error depends mainly on the discretization step value $h$. In fact, for a short rise time of dissipated power $\Delta P$, we can assume that the temperature value $T_{1}$ does not increase significantly for an important step value $h$. So, the introduced temperature error $\Delta T_{0}$ on temperature response $T_{0}$ is given by (4)

$$
\Delta T_{0}=\frac{h}{K S} \Delta P \text {. }
$$

Fig. 3(a) and (b) shows $T_{0}$ responses induced by a highpower pulse for different $h$ values. The parameters are $K=$ $1.54 \mathrm{~W} / \mathrm{cm} / \mathrm{K}, \rho c=1.63 \mathrm{~J} / \mathrm{cm}^{3} / \mathrm{K}, L=550 \mu \mathrm{m}$, and $S=10 \mathrm{~mm}^{2}$.

Using 50 nodes, the introduced temperature error $\Delta T_{0}$ is around $22 \mathrm{~K}$ (in the first instants corresponding to the establishment of the dissipated power to its maximum). To minimize this error, it should be used a very fine mesh in the proximity of the dissipated power source, which implies an important node number. The important temperature error $\Delta T_{0}$ (proportional to dissipated power variation) is due to the heat equation that is not satisfied for node number $i=0$. Finally, the boundary condition (4) is used in place of (3). Obviously, if a fine mesh is used $(n=1000)$, the numerical solution becomes near the solution of the boundary value problem.

\section{Finite-ElEMENT MethoD}

Another classical method used to develop thermal models is the FEM, which is based on a variational approximation [7] of the heat equation.

The approximated solution of (1) is given by

$$
T(x, t)=\sum_{i=1}^{m} \xi_{i}(t) W_{i}(x)
$$

where $W_{i}(x)$ are the decomposition functions and $\xi_{i}(t)$ are the coordinates of the temperature approximation in the functional space basis formed by the decomposition functions.

A trial function $S_{i}(x)$ is considered. Using (2a), the integration of (1) multiplied by a function $S_{i}(x)$ over $[0, L]$ yields the following variational equation associated to the boundary problem (1) and (2) [6]:

$$
\begin{gathered}
\frac{d}{d t} \int_{0}^{L} T(x, t) S_{i}(x) d x+\frac{K}{\rho c} \int_{0}^{L} \frac{\partial T(x, t)}{\partial x} \frac{d S_{i}}{d x} \\
-\frac{K}{\rho c} S_{i}(L) \frac{\partial T}{\partial x}(L, t)=\frac{P(t)}{\rho c S} S_{i}(0)
\end{gathered}
$$

where $S_{i}(x)$ are the trial functions. Classically, the boundary conditions (2) are taken into account in the variational equation (7). The internal approximation is the projection of the exact solution of the boundary value problem on the decomposition functional space. It is obtained by substituting (6) into (7).

Equation (7) applied for $m$ trial function $S_{i}(x)$ may be written in a matrix form (8)

$$
\frac{d X}{d t}=A X+B u
$$

where

$$
\begin{aligned}
X & =M \xi, A=-R M^{-1}, u=P_{\text {in }}(t) \text { and } \\
M_{i j} & =\int_{0}^{L} W_{j}(x) S_{i}(x) d x \\
R_{i j} & =\frac{K}{\rho c} \int_{0}^{L} \frac{d W_{j}}{d x} \frac{d S_{i}}{d x} d x-\frac{K}{\rho c} \frac{d W_{i}(L)}{d x} S_{i}(L) \\
B_{i} & =\frac{S_{i}(0)}{\rho c S}
\end{aligned}
$$




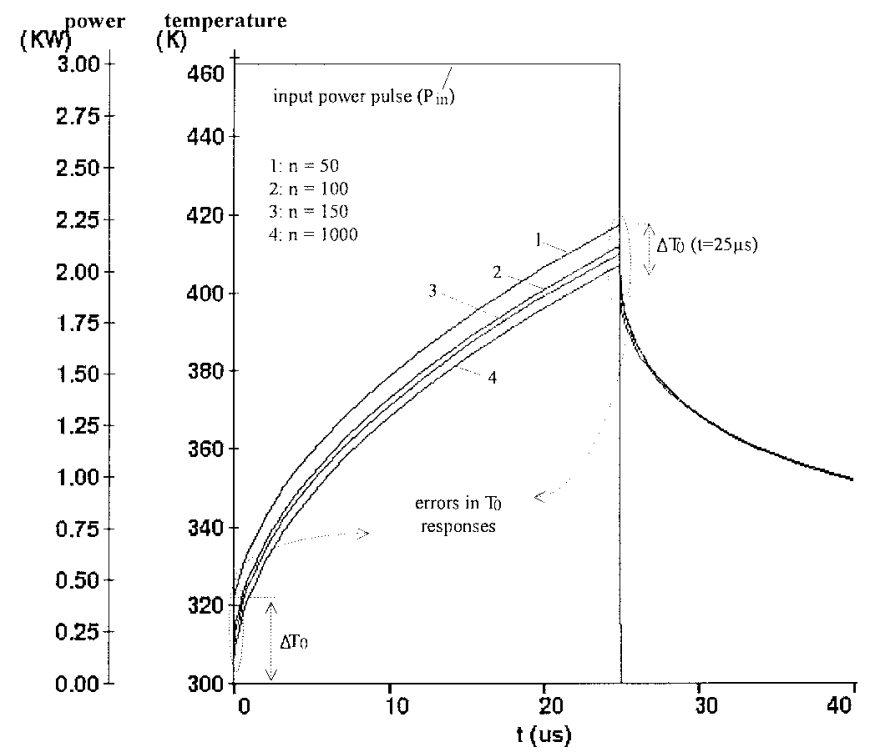

(a)

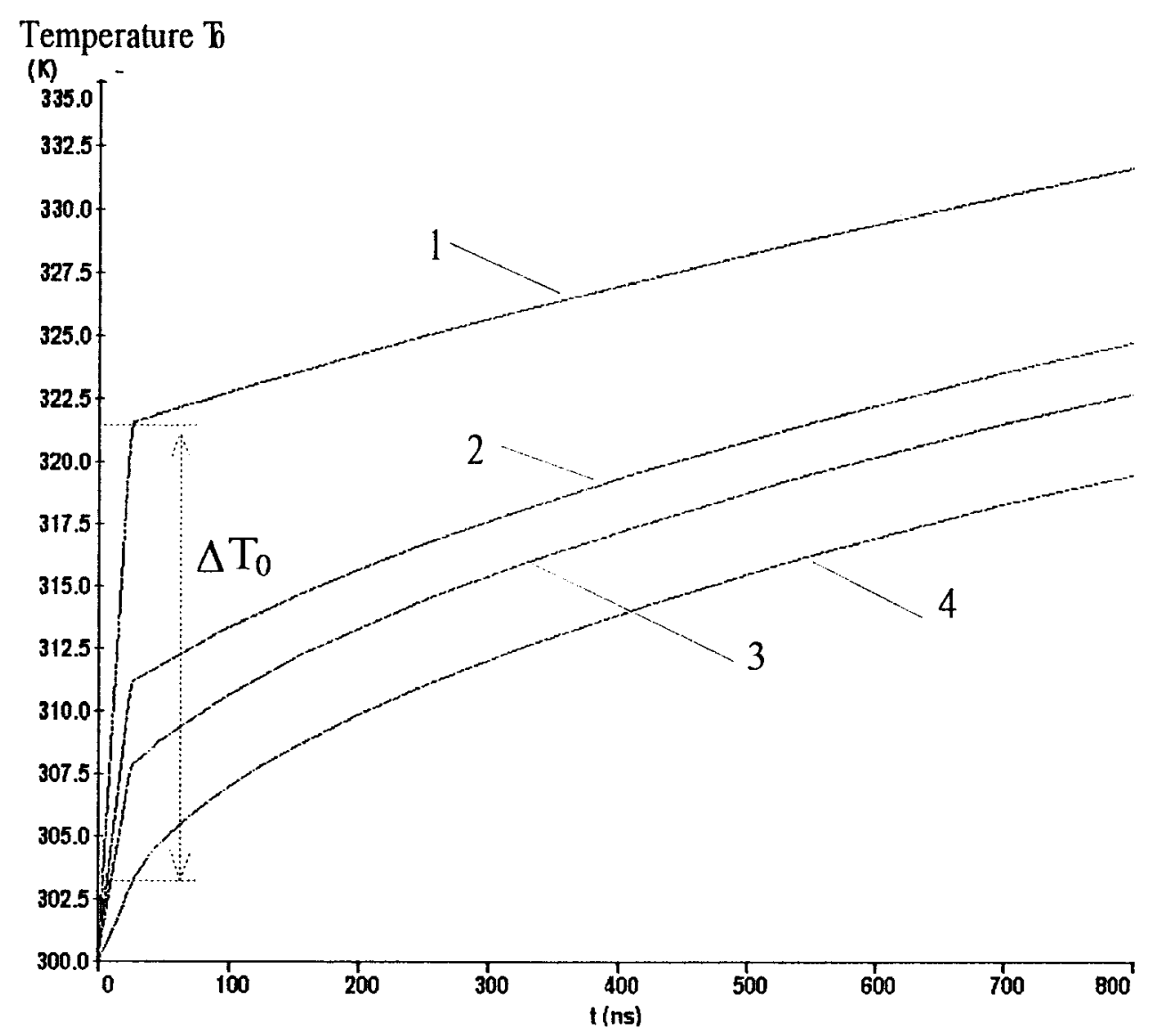

(b)

Fig. 3. (a) $T_{0}$ temperature responses obtained by the FDM for a different number of nodes. (b) Zoom of $T_{0}$ temperature responses obtained by the FDM.

So, the obtained model is a state-space model, where $X_{i}(t)$ are the state variables. For instance, an output value $y(t)$ of the state-space model may be the temperature at $x=0$

$$
y(t)=T(0, t)=W(0) M^{-1} X(t)
$$

The temperature $T(x, t)$ at abscissa $x$ is given by

$$
T(x, t)=W(x) M^{-1} X(t)
$$

where $W(x)$ is the row vector formed by the decomposition functions $W_{i}(x)$. 


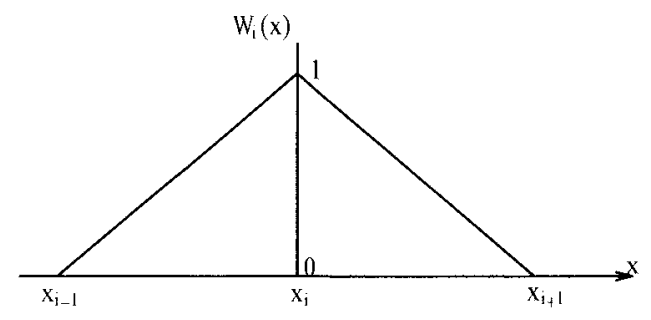

Fig. 4. Distribution function $W_{i}(x)$ used in the FEM.

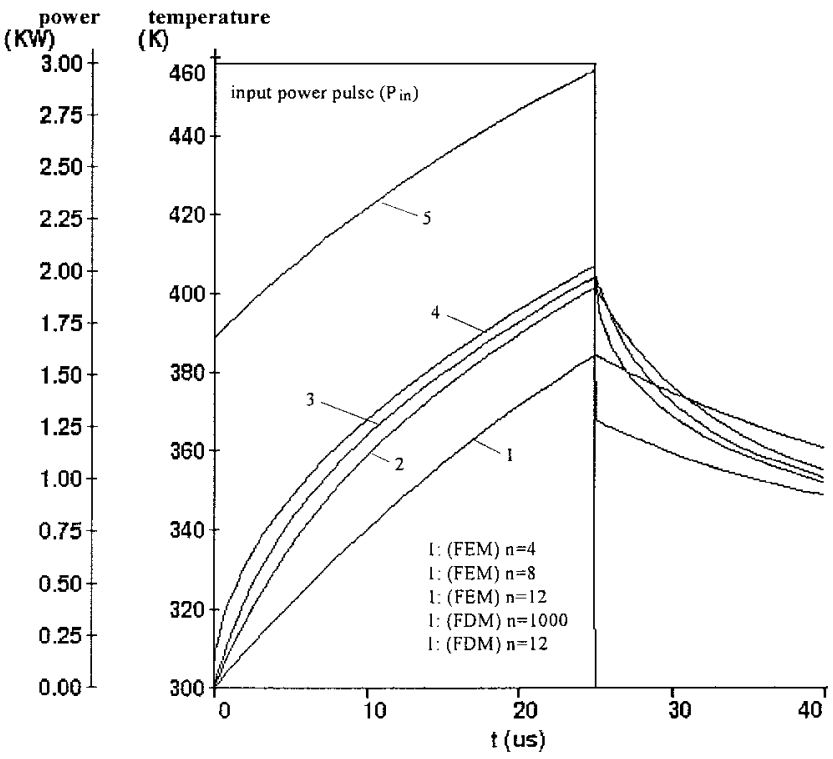

Fig. 5. $T_{0}$ temperature responses obtained by the FEM for different number of nodes.

Particularly, the FEM consists of a classical choice of the decomposition function $W_{i}(x)$, which is a linear piecewise function equal to one at node number $i$ and zero at any other node (Fig. 4). The advantage of this choice of $W_{i}(x)$ functions is the analytical calculation of matrix elements. Since the support of the function $W_{i}(x)$ (the set of $x$ satisfying $W_{i}(x) \neq$ 0 ) is a small part of the studied space $(0<x<L)$, the obtained matrix A is sparse because most of the matrix element corresponds to calculation of the integral of function $W_{i}(x)$ outside of their support. On the other hand, this choice can lead the temperature's coordinate $\xi_{i}(t)$ to correspond to the temperature itself or its derivative at node $(i)$. In our case, $\xi_{i}(t)$ correspond to the node temperature values. Classically, the trial functions $S_{i}(x)$ are chosen to be equal to the decomposition functions $W_{i}(x)$. This hypothesis simplifies the matrix configuration and the matrix A becomes symmetric. So, all of these remarks show that the FEM corresponds to a good choice of $W_{i}(x)$ function for an intensive numerical calculation. The equivalent circuit of the obtained 1-D thermal model is represented in Fig. 2 [5], where $C_{1}=(\rho c S h / 2), C_{2}=-(\rho c S h / 6)$, and $R_{1}=(h / K S)$.

Fig. 5 shows $T_{0}$ thermal responses given by the equivalent thermal circuit obtained by the FEM for different element numbers. The temperature $T_{0}$ has a smooth response corresponding to the step of dissipated power because of the capacitance $C_{1}$ in parallel connection with the power source.
TABLE I

ERror EVAluation of $T_{0}$ Responses $\left(\Delta T_{0}\right.$ Is tHE ERROR INTRODUCED IN $T_{0}$ RESPONSE)

\begin{tabular}{l|c|c}
\hline model error & $\Delta T_{O}(t=30 n s)$ & $\begin{array}{c}\Delta T_{O}(t=25 u s) \\
(K)\end{array}$ \\
\hline FEM $(n=4)$ & -2.5 & -22 \\
\hline FEM $(n=12)$ & -1 & -3.5 \\
\hline FDM $(n=12)$ & 88 & 60 \\
\hline FDM $(n=100)$ & 7.5 & 5 \\
\hline
\end{tabular}

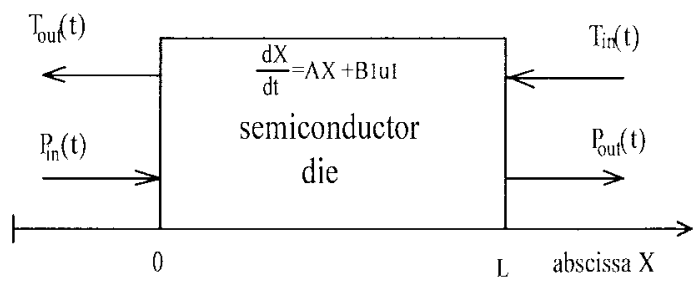

Fig. 6. The schematic representation of the input-output variables on the semiconductor die.

In fact, in the FEM the boundary conditions as well as the heat equations are completely included in variational equation (7). Fig. 5 shows clearly that the FEM gives a better thermal response than the FDM (Table I). The inconvenience of the FEM equivalent circuit is the negative value of the capacitance $C_{2}$ that corresponds to no physical meaning.

\section{ANALYTICAL Model AND INTERNAL APPROXIMATION}

The FEM is based on the choice of compact support functions. So, the classical choice of the decomposition functions $W_{i}(x)$ mentioned above supposes that the temperature distributions between the nodes in the device have the same evolution. These functions may correspond to a laser spot response, but not correspond to a physical description of the temperature evolution when the dissipated power is generated particularly at the top of the device.

Subsequently, the idea is to develop an efficient analytical model based on an internal approximation where the decomposition functions have a support equal to the complete physical domain $\left[W_{i}(x)\right.$ defined for $\left.0<x<L\right]$ instead of restriction of a neighborhood segment of a given node as in FEM.

The approximated solution of (1) may be written as

$$
T_{I A}(x, t)=T_{\mathbf{i n}}(t)+\sum_{i=1}^{m} \xi_{i}(t) W_{i}(x) .
$$

In this case, $\xi_{i}(t)$ does not correspond to the node temperature value. The variational equation associated with the boundary value problem [see (1)-(2b)] is given by (7) [6].

As developed in [6], the suitable choice of decomposition functions $W_{i}(x)$ enables a good approximation of the problem solution.

$P_{\mathrm{in}}(t)$ and $T_{\mathrm{in}}(t)$ are the input variables because the boundary value problem [see (1)-(2b)] depends on their values.

Consequently, $P_{\text {out }}(t)$ and $T_{\text {out }}(t)$ are output variables of the models. 


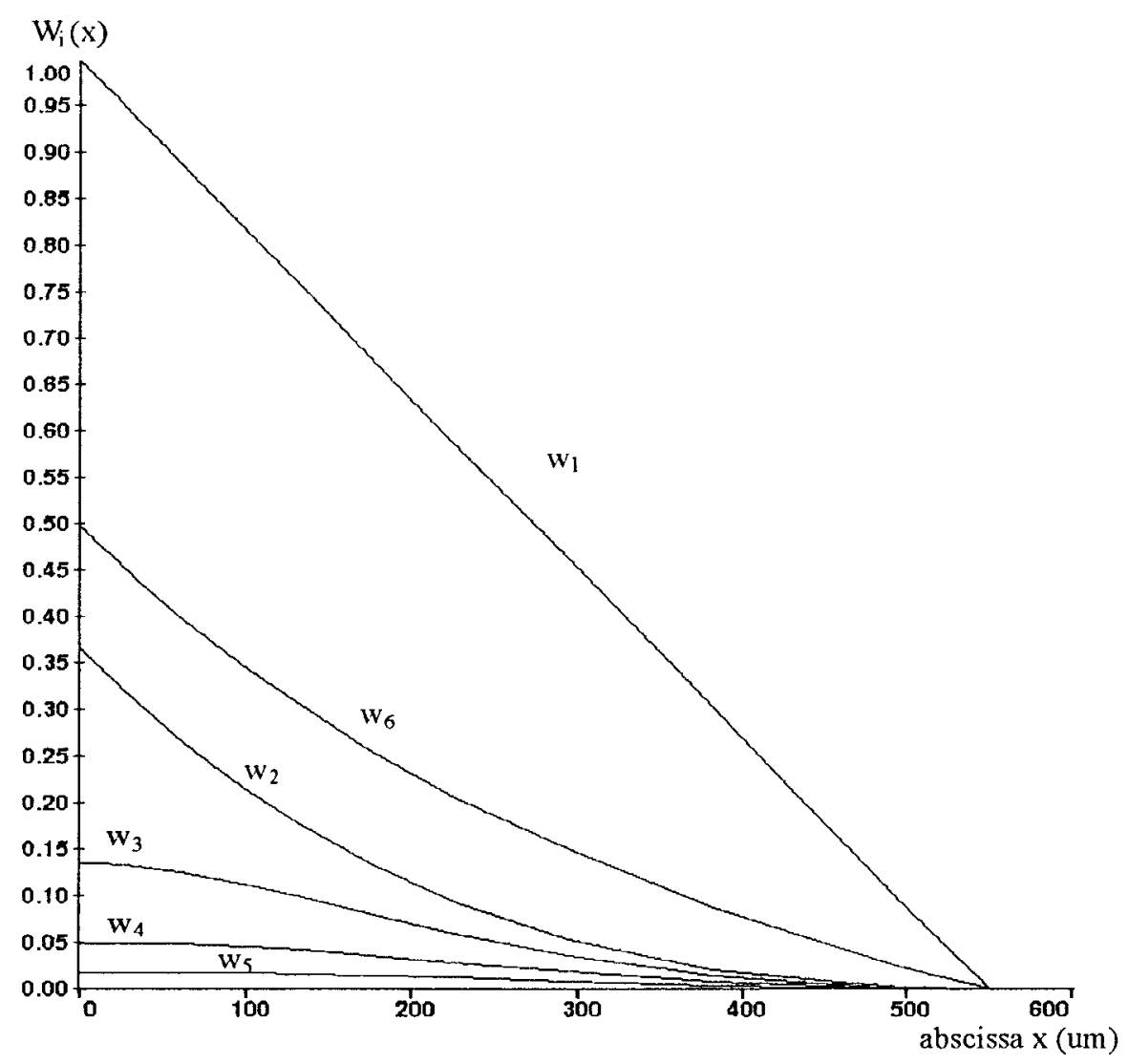

Fig. 7. Decomposition functions evolution $W_{i}(x)$.

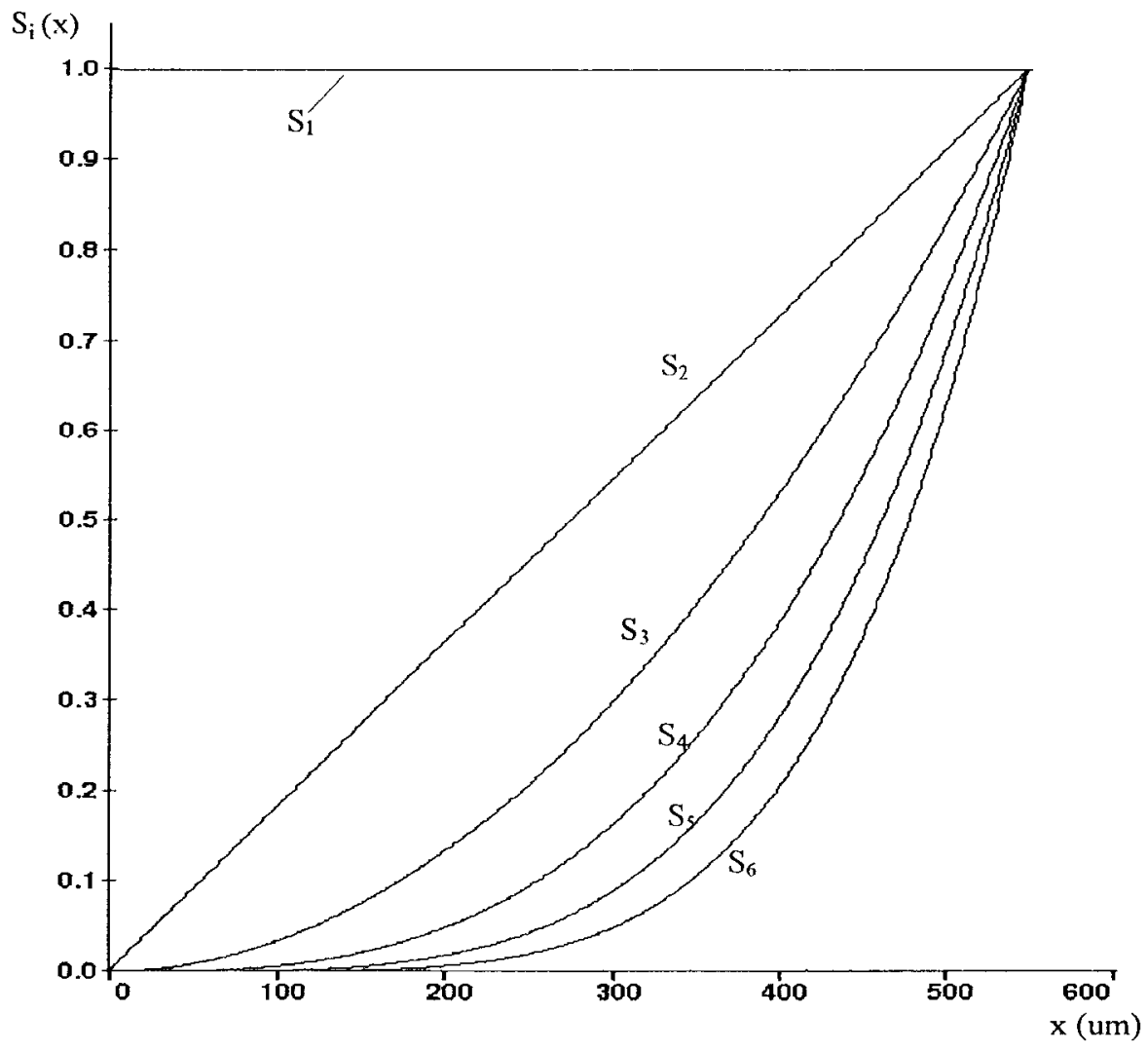

Fig. 8. The trial function evolution. 
The schematic representation of the input-output variables on the semiconductor die $(0<x<L)$ is represented in Fig. 6 [8].

As developed below (7) and taking into account (10), we obtain the following differential equations that represent the system dynamics:

$$
M \xi+R \xi=B P_{\text {in }}+C \frac{d T_{\text {in }}}{d t} .
$$

$C$ is a vector defined by

$$
C_{i}=-\int_{0}^{L} S_{i}(x) d x
$$

The state variable vector is defined as

$$
X=-M \xi+C T_{\mathrm{in}} .
$$

So, the state equation of the internal approximation of the boundary value problem [see (1)-(2b)] may be written as

$$
\frac{d X}{d t}=A X+B_{1} u_{1}
$$

where

$$
A=-R M^{-1} \text {. }
$$

$B_{1}$ is a two-column $/ m$-row matrix including the vector $(-B)$ and $(-A C)$

$$
B_{1}=(-B,-A C)
$$

and

$$
u_{1}=\left(\begin{array}{c}
P_{\mathrm{in}}(t) \\
T_{\mathrm{in}}(t)
\end{array}\right)
$$

The output relations are defined as

$$
\begin{aligned}
T_{\text {out }}(t) & =T_{\mathrm{in}}(t)+W(x=L) M^{-1}\left(C T_{\mathrm{in}}-X(t)\right) \\
P_{\text {out }}(t) & =-S K \sum_{i=1}^{m} \xi_{i}(t) \frac{\partial W}{\partial x}(x=L) \\
& =S K M^{-1} \frac{d W}{d x}(x=L)\left(X(t)-C T_{\mathrm{in}}\right) .
\end{aligned}
$$

In the case where the dissipated power is located at the top of the chip, the decomposition functions can be chosen like (Fig. 7)

$$
\begin{aligned}
W_{1}(x)= & 1-x / L \\
W_{i}(x)= & -(x / L)^{i-1} \exp (-x(i-1) / L) \\
& +\exp (-(i-1)), \quad(i=2 \rightarrow 5) \\
W_{i}(x)= & \alpha_{i}+\frac{\beta_{i}}{1+\gamma_{i} x}, \quad(i>5)
\end{aligned}
$$

satisfying

$$
W_{i}(L)=0
$$

because

$$
T(L)=T_{\mathrm{in}}
$$

which represents a good approximation of the physical evolution of the temperature in the device as given in Fig. 7.

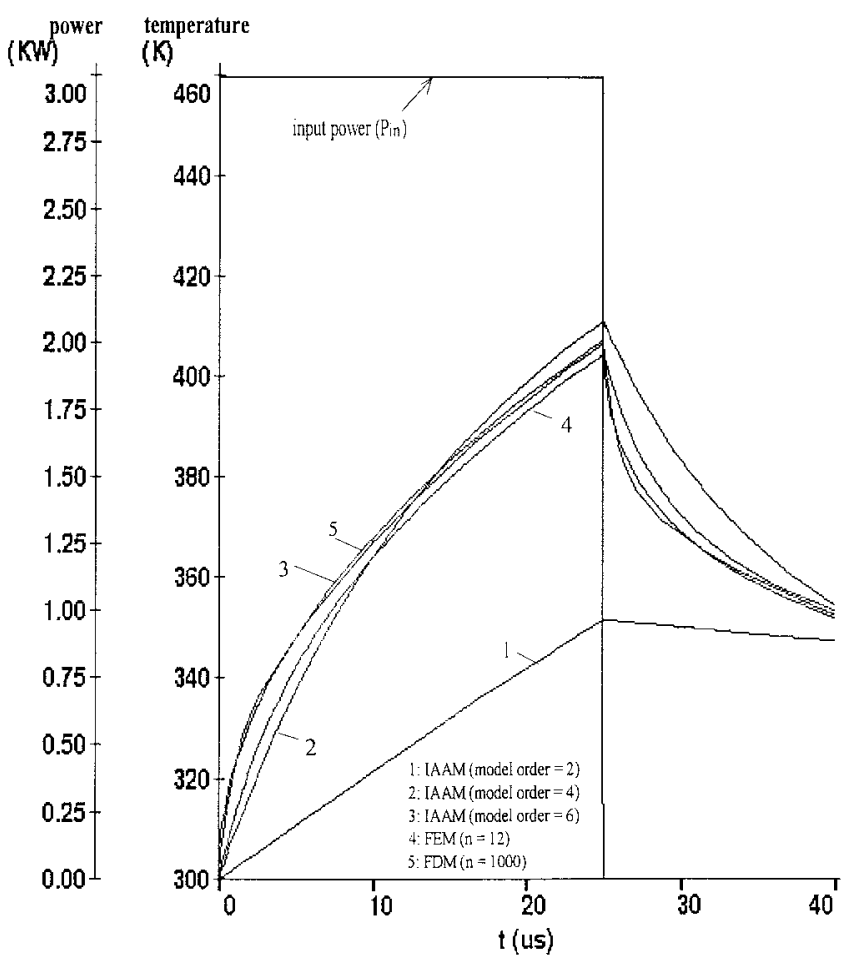

Fig. 9. $T_{0}$ temperature responses obtained by the IAAM for different order models.

The trial functions are chosen like (Fig. 8)

$$
S_{i}(x)=(x / L)^{i-1} \text {. }
$$

In fact, for $i=1, S_{1}(x)=1$ verifies the energy balance.

For $i \neq 1, S_{i}(x)$ are chosen so that the matrix $M^{-1}$ exists and the determinant of the matrix $M$ is large to avoid a numerical problem.

Fig. 9 gives simulation results obtained with model orders 2,4 , and 6 . In our case, $T_{\mathrm{in}}(t)$ is equal to room temperature $(300 \mathrm{~K})$ and $W_{6}(x)$ is defined as

$$
W_{6}(x)=-0.5+\frac{1}{1+x / L} .
$$

In the case of the sixth-order model, the numerical values of matrix $A$ and matrix $B_{1}$, obtained by Mathematica computations, are given in the equation given at the bottom of the next page and in

$$
B_{1}=-\frac{K}{\rho c L^{2}}\left(\begin{array}{cc}
L / K S & 27.805 \\
0 & 36.21 \\
0 & 30.84 \\
0 & 29.81 \\
0 & 32.84 \\
0 & 32.84
\end{array}\right) .
$$

We note that the IAAM model gives a good result for a model order larger or equal to six. This advantage compared to the FEM method is due to the choice of decomposition functions $W_{i}(x)$. In fact, in the IAAM model, $W_{i}(x)$ have a physical evolution that approaches the temperature distribution in the device. We note that this choice of $W_{i}(x)$ gives good results, concerning the temperature evolution, when the dissipated power is located at the top of the device. In other 


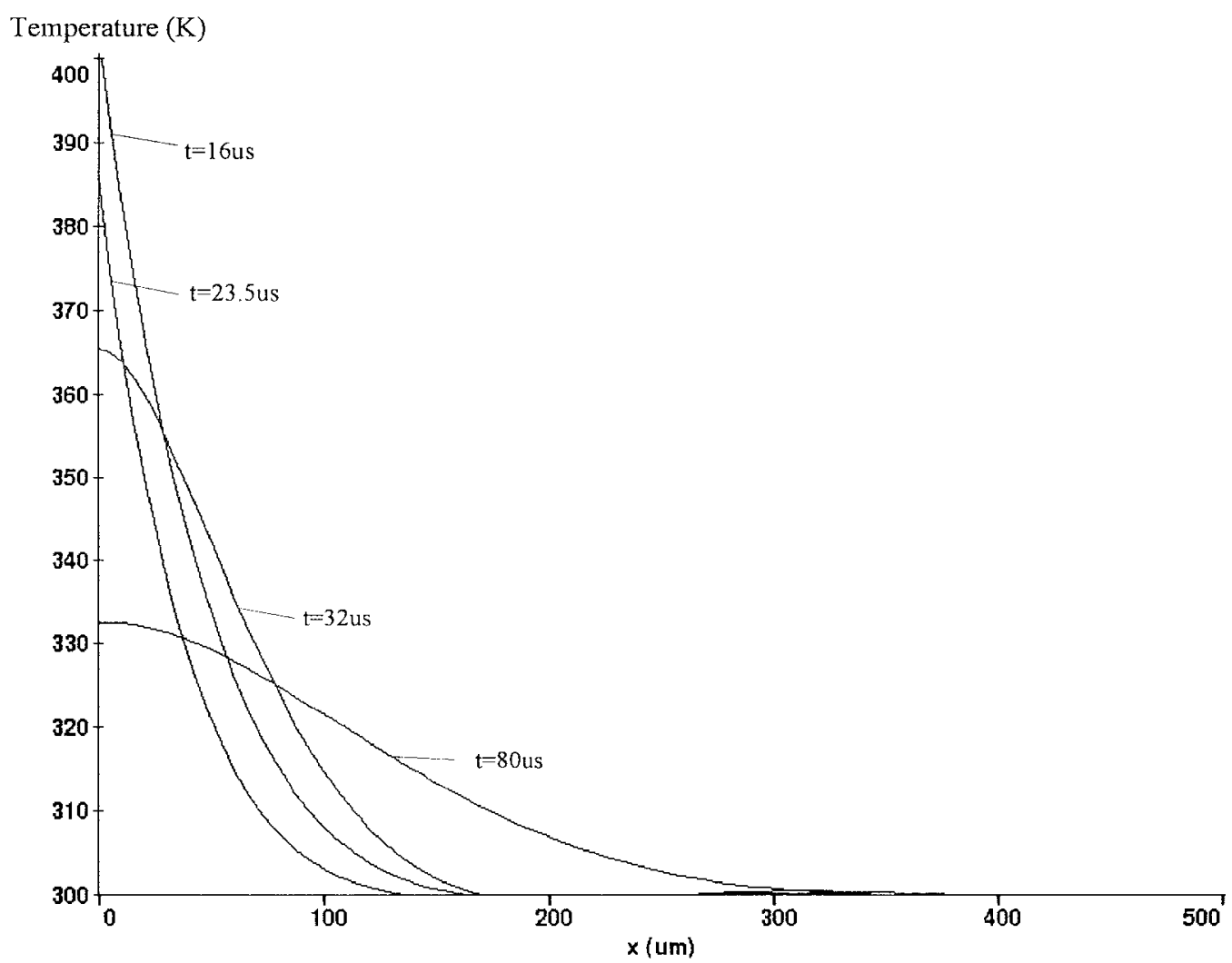

Fig. 10. Temperature distribution at different instants.

cases (dissipated power at the bottom or in the volume), another decomposition function evolution will be chosen. Fig. 10 shows the temperature evolution versus the abscissa $x$ for different instants. This distribution is in agreement with the decomposition function $W_{i}(x)$ evolution. So, we should have an idea about dissipated power locations and temperature evolution in the device to define the decomposition functions. We note that it is possible to discretize the device structure with the IAAM method, this discretization enables (for example) the introduction of dissipated power source in the volume.

The analytical model developed here is easy to implement in circuit simulators (ELDO [9], PACTE [10], SABER [11], SMASH [12], etc.) using high-level description language. The different IAAM models for orders 2, 4, and 6 have been developed using $M^{++}$language with a PACTE simulator and translated in ABCD for a SMASH simulator. Nevertheless, the translation into other simulator languages is possible. These models give good results with simulation central processing unit (CPU) times less than what is needed by the FEM method.

\section{CONCLUSION}

In this paper, we have presented a comparison between three 1-D thermal models (FDM, FEM, and IAAM) for electrothermal simulation of power semiconductor devices. We have studied especially the effect of the boundary condition representation at the position of the heat source. Because the discretized heat equation is not satisfied at the node corresponding to the boundary equation (2a), the FDM introduces an large error in temperature responses especially at the heat source. This error depends mainly on the discretization step value $h$ and the dissipated power step value per unit area. So, the FDM is not an accurate method for thermal modeling.

On the other hand, the FEM and the analytical model (IAAM) do not introduce such an error. They represent accurately the boundary condition at the heat source because this condition is included in the variational equation derived from the heat equation.

Finally, concerning thermal model circuit networks, it is clear that for electrothermal simulation of power semiconductor devices, the FEM [1-D, two-dimensional (2-D), and

$$
A=\frac{K}{\rho c L^{2}}\left(\begin{array}{cccccc}
8.655 & -428.262 & 4553.27 & -17331.5 & 26535.7 & -13916.1 \\
61.08 & -1668.68 & 13032 & -40834.2 & 54639.5 & -25957.2 \\
10.655 & -428.3262 & 4553.27 & -17331.5 & 26535.7 & -13916.1 \\
8.655 & -422.262 & 4553.27 & -17331.5 & 26535.7 & -13916.1 \\
8.655 & -428.262 & 4565.27 & -17331.5 & 26535.7 & -13916.1 \\
8.655 & -428.262 & 4553.27 & -17331.5 & 26535.7 & -13916.1
\end{array}\right)
$$


three-dimensional (3-D)] gives more accurate results than the FDM for the same number of nodes.

For an adequate choice of the decomposition function in the IAAM method, we obtain an interesting result with a model order less than the one used in the FEM method. We note that it is very difficult to formulate the IAAM method in the case of 2-D and 3-D models, and the good results given by this method are tributary of the idea that we have about the temperature distribution in the device a priori. For 2-D or 3-D thermal phenomena, the FEM model is easier to implement than the IAAM model. So, for multidimensional thermal phenomena, a mixed thermal model (FEM and IAAM models) enables to obtain good results in an optimized simulation time.

\section{REFERENCES}

[1] J. Gustavsen and W. Roehr, "Thermal response of semiconductors," MOTOROLA Semiconductor Products, Inc. Application Note (AN292), pp. 2-11.

[2] S. Clemente, "Transient thermal response of power semiconductors to short power pulses," IEEE Trans. Power Electron., vol. 8, pp. 337-341, Oct. 1993.

[3] A. R. Hefner and D. L. Blackburn, "Thermal component models for electrothermal network simulation," IEEE Trans. Comp., Packag. Manufact. Technol. A, vol. 17, pp. 413-424, Sept. 1994.

[4] P. R. Striskland, "The thermal equivalent circuit of a transistor," IBM J., pp. 35-45, Jan. 1959

[5] J. T. Hsu and L. Vu-Quoc, "A rational formulation of thermal circuit models for electrothermal simulation-Part I: Finite element method," IEEE Trans. Circuits Syst. I, vol. 43, pp. 721-732, Sept. 1996.

[6] A. Ammous, B. Allard, and H. Morel, "Transient temperature measurements and modeling of IGBT's under short-circuit," IEEE Trans. Power Electron., vol. 13, pp. 12-25, Jan. 1998.

[7] R. E. Shawalter, Hilbert Space Methods for Partial Differential Equation. New York: Pitman, 1977.

[8] H. Morel, S. H. Gamal, and J. P. Chante, "State variable modeling of the power pin diode using an explicit approximation of semiconductor device equations: A novel approach," IEEE Trans. Power Electron., vol 9, no. 1, pp. 112-120, 1994

[9] Eldo, HDL-A User's Manual, ANACAD Electrical Engineering Software, France, July 1994.

[10] B. Allard, H. Morel, S. Ghedira, and A. Ammous, "A bond graph simulator for power train simulation including semiconductor device model," in IEEE Proc., IMACS' 96, Lille, France, pp. 500-505.

[11] Saber, Mast Reference Manual, Analogy, Inc., Beaverton, OR, Oct. 1996.

[12] SMASH, ABCD Language Reference Manual, Dolphin Integration, Meylan Cedex, France, Apr. 1996.

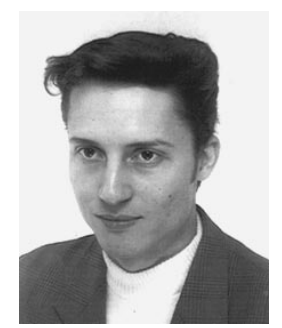

Anis Ammous was born in Sfax, Tunisia, on April 4, 1970. He received the electrical engineering degree from the Ecole National d'Ingenieur de Sfax (ENIS), Sfax, in 1994, the Diplome des Etudes Approfondies (DEA) degree in power electronics from the Institut National Polytechnique de Toulouse (INPT), Toulouse, France, in 1995, and the Ph.D. degree in electrical engineering from the Institut National des Sciences Appliquees (INSA), Lyon, France, in 1998.

His current research interests are power semiconductor device modeling and the electrothermal modelization and failure studies of IGBT's.
Sami Ghedira was born in Monastir, Tunisia, on April 12, 1967. He received the Master of Science degree from the University of Monastir, Tunisia, in 1993 and the Diplome des Etudes Approfondies (DEA) and the Ph.D. degrees in integrated electronics from the Institut National des Sciences Appliquees (INSA), Lyon, France, in 1994 and 1998, respectively.

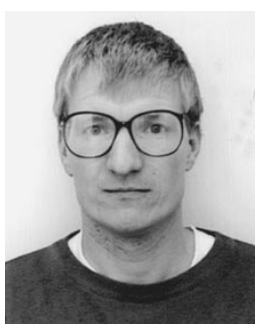

Bruno Allard (M'92) received the electrical engineering, M.Sc., and Ph.D. degrees from the Institut National des Sciences Appliquees (INSA), Lyon, France, in 1988, 1989, and 1992, respectively.

From 1991 to 1993 , he was with the INSA as an Associate Professor. Since 1993, he has been an Assistant Professor. His present research interests include power semiconductor device modeling, power system simulation, and applications of bond graph to power electronics.

Dr. Allard is a member of the European Power Electronic Society and the European Working Group of the IEEE Industrial Applications Society.

Hervé Morel was born in Reims, France, in 1959. He received the electrical engineering and Ph.D. degrees from Ecole Centrale de Lyon, Lyon, France, in 1982 and 1985 , respectively.

In 1986, he joined the Centre National de la Recherche Scientifique (CNRS), Lyon, where he was engaged in research on power electronic system modeling in the Centre de Genie Electrique de Lyon (CEGELY), Lyon. His research areas include power semiconductor device modeling and multidomain modeling of power electronic systems based on bond graph. He was involved with the coordination of the CAD Tool for Automotive Electronics for the French contribution of the European project PROCHIP

Dr. Morel is a member of the Society of Computer Simulation.

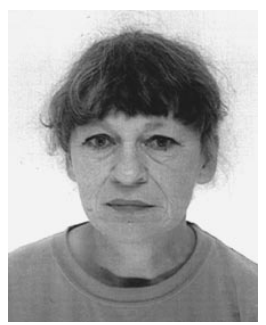

Denise Renault received the cybernetic engineering degree from ISIN, Nancy, France, in 1966.

She is currently a System Engineer at the laboratory of the Centre de Genie Electrique de Lyon (CEGELY), Lyon, France. She is the System Administrator for all the designing tools such as $\mathrm{Ca}$ dence, ISE, and simulation tools such as SILVACO (WMF), TMA, etc. She also provides software assistance for the researchers. 